\title{
Anomalie héréditaire de l'expression des molécules HLA de classe II
}

Le défaut d'expression des antigènes de classe II du complexe majeur d'histocompatibilité entraîne un déficit immunitaire sévère. Son mécanisme pourrait bien constituer le premier exemple connu en pathologie humaine de la mutation d'un gène régulateur agissant en trans.

\section{Barbara Lisowska- Grospierre Chargee de Recherche à l'Inserm}

Claude Griscelli

Directeur de l'Unité Inserm U. 132

\section{RÉFÉRENCES}

1. Benaceraff B. In : Dorf ME, ed. The role of the Major Histocompatibility Complex in Immunobiology. New York, Garland, 1981 ; 255 p.

2. Schwartz RH. The role of gene products of the MHC in T cell activation and cellular interactions. In : Paul WE, ed. Fundamental Immunology. New York : Raven Press, 1984 : 379-439.

\section{ADRESSE}

B. Lisowska-Grospierre, C. Griscelli : Inserm, U. 132, hôpital Necker-Enfants Malades, 149, rue de Sèvres, 75015 Paris.

$\mathrm{m} / \mathrm{s} n^{\circ} 1$ vol. 3 , janvier 87 'expression membranaire des antigènes d'histocompatibilité joue un rôle essentiel dans les phénomènes de reconnaissance des antigènes. Les molécules de classe I (HLA A, B et $\mathrm{C}$ ), comme les molécules de classe II (DR, DQ DP) interviennent dans ces phénomènes. La présentation conjointe des molécules HLA de classe II et de l'antigène (au niveau de la membrane des cellules présentatrices de l'antigène) est nécessaire à toute réponse immunitaire [1-3]. Les gènes du complexe majeur d'histocompatibilité (CMH) sont situés, chez l'homme, sur le bras court du chromosome 6 . Les molécules HLA de classe I sont exprimées sur toutes les cellules nucléées et sont composées d'une chaîne lourde de $44 \mathrm{Kd}$ associée à une chaîne légère de $12 \mathrm{Kd}$, la Bêta microglobuline $(\beta 2 \mathrm{~m})$ dont le gène est situé sur le chromosome 15 [4]. Les molécules HLA de classe II sont exprimées à la membrane des lymphocytes $\mathrm{B}$, des monocytes-macrophages et des lymphocytes $\mathrm{T}$ activés. Ces molécules sont également exprimées à la membrane des cellules endothé- liales, des cellules de Langerhans et des cellules épithéliales du thymus et de l'intestin ; ce sont des dimères composés d'une chaîne $\alpha$ de $33-34 \mathrm{Kd}$ et d'une chaîne $\beta$ de $28-29 \mathrm{Kd}$ qui sont associées au niveau intracytoplasmique à un troisième peptide de $31 \mathrm{kd}$ appelé chaîne invariante (Inv). La chaîne Inv n'est pas exprimée au niveau de la membrane. Son rôle n'est pas encore défini avec certitude mais il est probable qu'elle intervient dans l'assemblage et/ou le transport des molécules dimériques $(\alpha-\beta)$ vers la membrane. L'organisation des molécules HLA dans la membrane (schématisée dans la (figure 1) comprend, pour chaque chaîne $\alpha$ et $\beta$, des molécules HLA de classe II, deux domaines extracytoplasmiques $\left(\beta_{1} \beta_{2}\right.$ et $\alpha_{1}$ $\alpha_{2}$ ), un domaine transmembranaire et un domaine intracytoplasmique [5]. Dans les phénomènes de reconnaissance de l'antigène (figure 2), le polymorphisme extrême des molécules HLA et des molécules du récepteur $\mathrm{T}$ paraît essentiel pour assurer une affinité sélective vis-à-vis des différents antigènes. Le polymorphisme allélique de ces molécules 


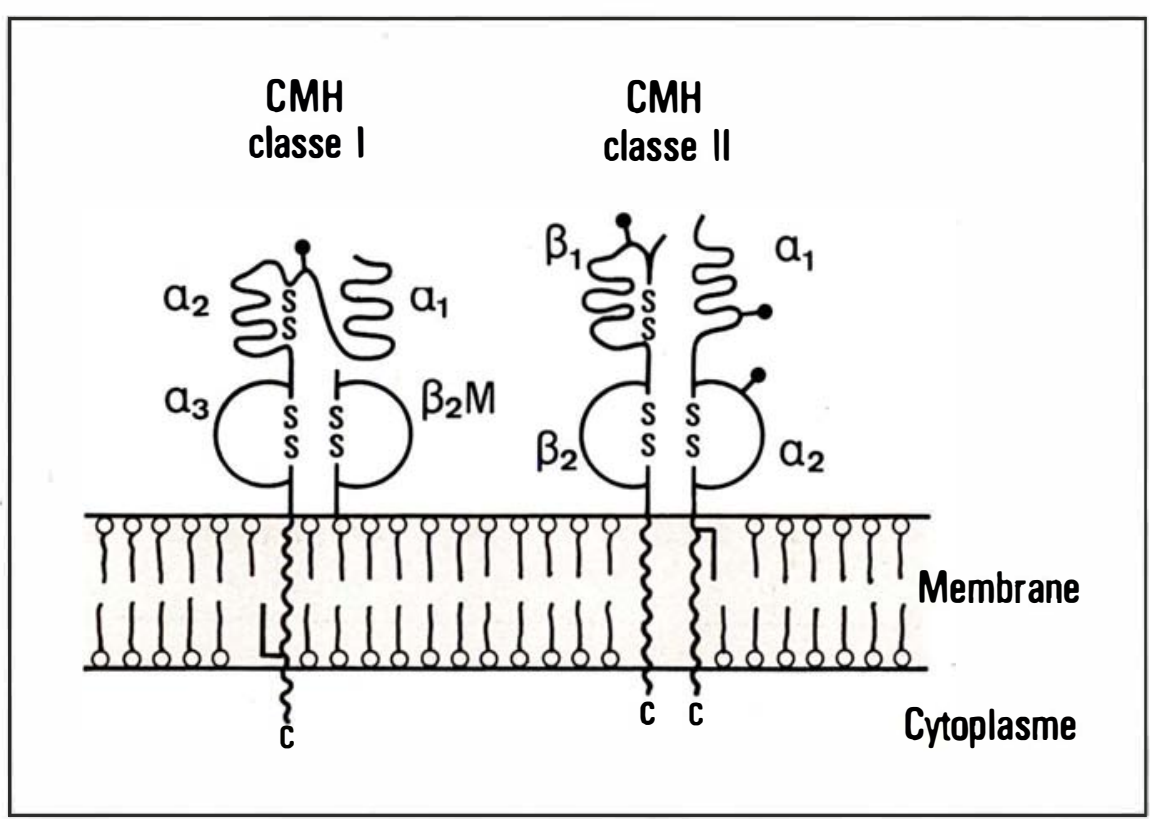

Figure 1. Structuro ot organisation des molécules HLA do classe I ot do classe II au niveau de la membrane cellulaire. S-S = ponts disulfures, $C$ $=C$-terminaux, ( $)$ ) chaînes d'hydrate de carbone. D'après [5].

a été reconnu depuis le début des études sérologiques. Ces dernières années, l'introduction des techniques de génétique moléculaire a permis l'étude de la structure et de l'organisation des gènes du $\mathrm{CMH} ; 14$ gènes HLA de classe II ont ainsi été reconus (figure 3), la plupart étant impliqués dans la synthèse des molécules HLA de classe II [6]. Les mécanismes de régulation de l'expression des gènes des HLA de classe I et de classe II sont donc certainement complexes et restent encore mal connus. On sait en effet que : (a) l'expression des molécules HLA de classe II varie en fonction des étapes de la différenciation et de la maturation des cellules, particulièrement au niveau de la lignée lymphocytaire $\mathrm{T}[5]$; (b) l'activation des lymphocytes $T$ entraîne l'apparition membranaire des molécules HLA de classe II, du récepteur de

teur pour l'interleukine 2 (TAC) [5] ; (c) tous les interférons $(\alpha, \beta$ et $\gamma)$ sont capables d'augmenter l'expression des molécules HLA de classe I, l'interféron $\gamma$ augmentant aussi celle des molécules HLA de classe II. Cet interféron est un inducteur puissant puisqu'il est capable de faire apparaître les molécules HLA de classe II à la membrane des fibroblastes qui, normalement, n'expriment pas ces molécules [7].

Les études fonctionnelles reposent essentiellement chez l'homme sur des expériences réalisées in vitro. Elles comportent principalement l'analyse des effets exercés par différents anticorps anti-HLA sur les diverses fonctions immunologiques. La description d'une nouvelle maladie génétique caractérisée par un défaut de synthèse des molécules HLA de classe II et une diminution de l'expression membranaire des molécules HLA de classe I est donc d'un très grand intérêt. Elle permet de confirmer le rôle essentiel de l'expression membranaire des molécules d'histocompatibilité dans les phénomènes de reconnaissance de l'antigène et également de suggérer très fortement l'existence de gène(s) impliqué(s) dans la régulation de l'expression des gènes HLA de classe II.

\section{Le déficit immunitaire}

L'affection provoquée par un défaut de synthèse des molécules HLA de classe II fut identifiée pour la première fois en 1980 [8]. Une autre maladie, appelée syndrome des lymphocytes dénudés (Bare lymphocyte syndrome) [11] et caractérisée par un défaut d'expression membranaire des molécules HLA de classe I, avait été décrite en 1978 par Touraine et coll. [9] et par Schuurman et coll. [10]. Le déficit immunitaire associé à un défaut de synthèse des molécules HLA de classe II (MHC classe II-négative SCID, selon la nomenclature de l'OMS) est une affection transmise selon le mode autosomique récessif. La haute fréquence de consanguinités (9 mariages entre apparentés sur 14 familles étudiées, le nombre total d'enfants atteints étant de 17) suggère que la mutation est rare. Les premières infections apparaissent très tôt dans la vie, généralement avant le sixième mois. Elles sont caractérisées par l'association d'infections intestinales et bronchiques. La diarrhée devient chronique et engendre un retard staturo-pondéral important. Les infections bronchopulmonaires sont sévères. Il existe une susceptibilité particulière aux infections virales provoquées tant par les virus herpès et les adénovirus que par des entérovirus, responsables quant à eux de méningo-encéphalites. Parmi les autres manifestations, on observe fréquemment des cytopénies autoimmunes et une atteinte hépatique liée à une angiocolite sclérosante, probablement secondaire aux troubles digestifs chroniques. Dans notre série, l'expression clinique est voisine de celle 
des cas décrits par Haddam et coll. [12] et par Kuis et coll. [13]. Les anomalies immunologiques observées dans ce déficit immunitaire sont particulières. Les lymphocytes $\mathrm{T}$ et $\mathrm{B}$ sont présents en quantité normale dans le sang et les organes lymphoïdes mais sont fonctionnellement anormaux, les réponses cellulaires et humorales vis-à-vis des antigènes étant perturbées. La négativité des réactions cutanées d'hypersensibilité retardée est correlée, in vitro, à un défaut de prolifération lymphoblastique en présence de divers antigènes. Ces anomalies contrastent avec des capacités conservées de prolifération lymphoblastique en présence de mitogènes ou de cellules allogéniques. Les taux sériques d'immunoglobulines sont variables d'un malade à l'autre, mais la production d'anticorps

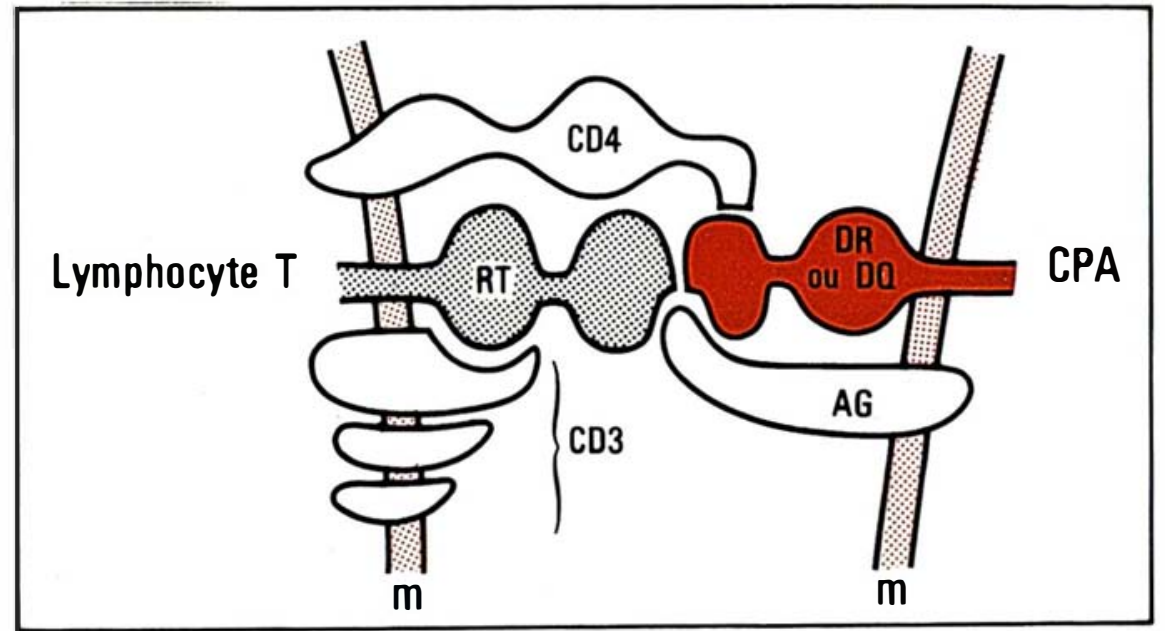

Figure 2. Schéma do la reconnaissanco do l'antigèno. Selon la conception actuelle, le récepteur $T$ (RT) reconnaît l'antigène (AG) au niveau de la membrane $(m)$ des cellules présentatrices de l'antigène (CPA) en association avec les molécules HLA de classe II IDR ou DQ, ceci n'étant pas encore démontré pour DPI. Les molécules CD3 et les molécules CD4 interviennent dans ces phénomènes complexes.

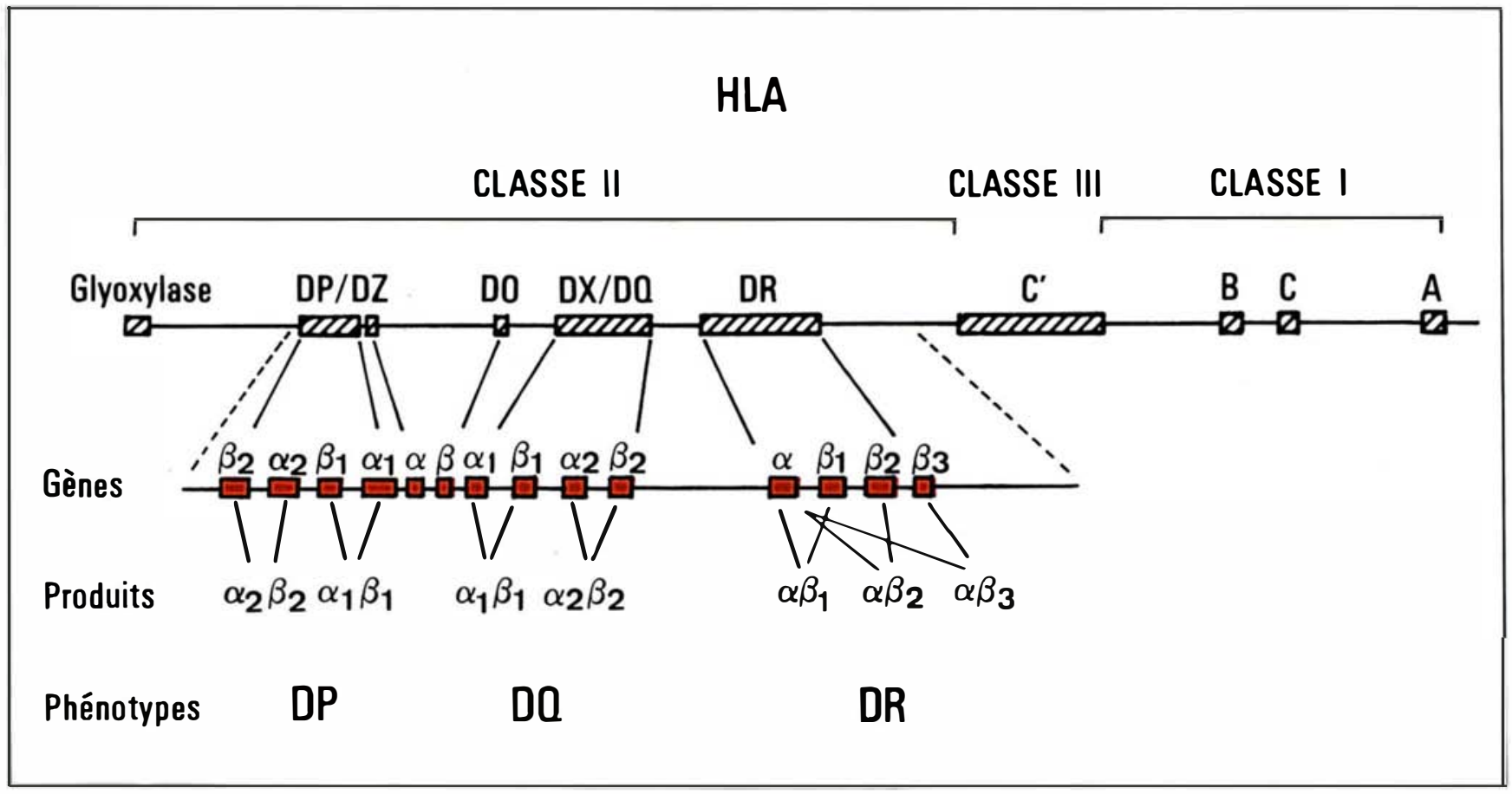

Figure 3. Gènes do la région CMH de classe II ot lours produits (modifié d'après FH Bach, Immunology Today, $1985, n^{\circ} 3$, volume 6 , p. 89-94).

$\mathrm{m} / \mathrm{s} n^{\circ} 1$ vol. 3 , januier 87 
après vaccination par divers antigènes est toujours très perturbée. Ceci suggère que les mécanismes d'activation cellulaire sont normaux et que les lymphocytes $B$ de ces malades sont capables de produire des immunoglobulines sous l'influence de facteurs d'activation polyclonaux comme les endotoxines bactériennes. Il faut aussi préciser que, chez certains malades, des anticorps d'isotype IgM (allohémagglutinines de groupe sanguin et anticorps antipolysaccharide anti-mannane du Candida albicans) peuvent être détectés dans le sérum. Exceptionnellement, dans le cas d'une infection virale persistante comme l'infection à cytomégalovirus (CMV) avec excrétion virale continue, des anticorps anti-CMV sont également produits.

Diverses méthodes permettent de détecter aisément le défaut d'expression membranaire des antigènes d'histocompatibilité. Du fait de la non-expression des molécules HLA de classe II, le phénotypage classique par étude de la cytotoxicité, réalisé sur les populations leucocytaires sanguines totales ou isolées (lymphocytes B et monocytes) est impossible. En revanche, les molécules HLA de classe I sont souvent détectables par cette méthode [8]. L'utilisation, en immunofluorescence, d'anticorps monoclonaux permet de préciser que les lymphocytes $\mathrm{B}$ et les monocytes n'expriment pas les molécules HLA de classe II $[14,15]$.

D'autre part, la technique des cultures allogéniques permet de confirmer le défaut de stimulation des leucocytes normaux par les leucocytes des malades. Il est également démontré que les lymphoblastes induits in vitro en culture allogénique ou en présence de mitogènes sont incapables d'exprimer les HLA de classe II tandis qu'ils expriment les HLA de classe I et les molécules d'activation (T9 et TAC) [16]. Au niveau tissulaire, diverses études réalisées par N. Bensussan montrent que le défaut d'expression des molécules HLA de classe II affecte égale- vasculaire, les cellules épithéliales de l'intestin (entérocytes) et du thymus. Dans la peau, quelques cellules de Langerhans peuvent, chez certains malades, exprimer faiblement des molécules HLA de classe II.

Ces anomalies d'expression membranaire des molécules HLA de classe II ne peuvent être corrigées par incubation des cellules en présence d'interféron $\gamma$. En revanche, la diminution de l'expression des antigènes de classe I est totalement corrigée par l'incubation en présence d'interféron $\alpha, \beta$ ou $\gamma$ [14]. Cependant, l'anomalie membranaire persiste sur les lymphoblastes $\mathrm{T}$ ou $\mathrm{B}$ mis en présence de phytohémagglutinine (PHA) ou d'interleukine 2 (IL-2) et les lignées induites par le virus d'Epstein-Barr [16]. Nous avons pu également montrer que chez

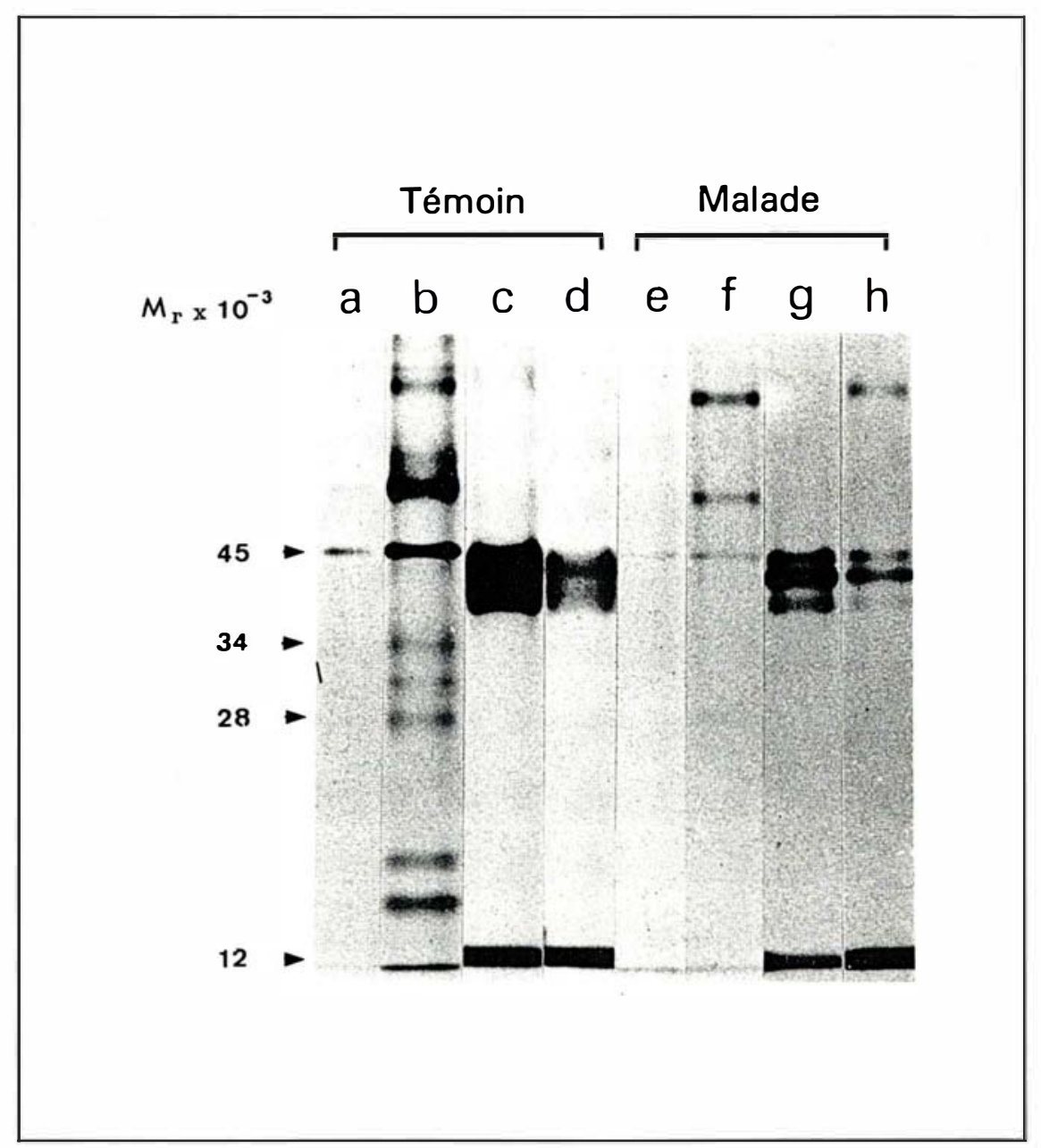

Figure 4. Analyso par électrophorèse on gol do polyacrylamido des próclpltés obtenus par les anticorps antl-classe II ot anti-classe I. Les lysats cellulaires sont obtenus à partir des blastes d'un malade et d'un sujet normal témoin. Les blastes sont induits par la PHA en présence de méthionine S35. Ils sont soumis à la précipitation par le sérum de souris normale (a, e), l'anticorps monoclonal (Mab) 206 anti-classe II (b, f), le Mab W6/32 anti-classe I (c, g), et l'anticorps de lapin anti $\beta 2$ microglubuline $(d, h)$. Les bandes ayant une migration apparente de 34 et 28 KD dans les blastes normaux (b) correspondent aux chaînes $\alpha$ et $\beta$ des HLA de classe II. Elles ne sont pas détectables chez le patient (f). La bande de $45 K D$ correspondant à la chaîne lourde des HLA de classe l et la bande de 12 KD correspondant à la $\beta 2$ microglobuline sont détectées chez le patient $(g, h)$. Leur quantité est inférieure à celle du sujet témoin (cd). 
des malades qui expriment une moindre quantité d'HLA de classe I à l'état basal, les lymphoblastes $\mathrm{T}$ (induits par la PHA) sont de très mauvaises cibles pour les cellules cytotoxiques. Néanmoins, leur traitement in vitro, en présence d'interféron, permet leur destruction [17].

\section{Le défaut} de biosynthèse

Le défaut d'expression membranaire des molécules HLA a tout d'abord été confirmé par marquage des cellules à l'iode 125 , suivi d'une précipitation des lysats en présence de divers anticorps monoclonaux dirigés contre les HLA de classe I (chaîne lourde et $\beta 2 \mathrm{~m}$ ) ou de classe II (chaîne $\alpha$ et $\beta$ ), et analyse des précipités par électrophorèse en gel de polyacrylamide. L'incorporation de méthionine $\mathrm{S}^{35}$ dans des lymphocytes ou des blastes induits par la PHA a permis de démontrer que les cellules des malades ne synthétisent pas de molécules HLA de classe II. L'anomalie d'expression membranaire est donc la conséquence d'un défaut de synthèse (figure 4). Ces mêmes expériences ont pu montrer que les cellules de ces patients contiennent une quantité réduite de molécules HLA de classe I [15, 17]. Il est important de préciser que la synthèse des molécules HLA de classe I est totalement corrigée par leur incubation préalable en présence d'interféron $\gamma$, témoignant du fait que ces cellules possèdent bien des récepteurs de ce type d'interféron. En revanche, la synthèse des molécules HLA de classe II n'est pas influencée par l'interféron $\gamma$ [17].

\section{Étude génétique}

La transmission du trait génétique de cette affection est indépendante du complexe majeur d'histocompatibilité. En effet, dans une étude réalisée dans plusieurs familles par C. Brémard-Oury, il apparaît que le trait génétique et le phénotype HLA ne coségrègent pas. Dans l'exemple $m / s n^{\circ} 1$ vol. 3, janvier 87 montré figure 5, deux sujets atteints sont HLA différents, et les jumeaux sains de la fratrie sont HLA identiques à l'un des deux patients. L'absence d'anomalie au niveau des gènes de structure de classe II du CMH a été confirmée par Marcadet et coll. [18] qui montrent, par analyse en Southern blot utilisant des sondes correspondant aux gènes HLA de classe II, qu'aucune anomalie grossière n'est détectable. Parmi les autres hypothèses, figurait l'idée d'un défaut de synthèse de la chaîne invariante supposée intervenir au niveau de l'assemblage et de la migration intracellulaire des chaînes $\alpha$ et $\beta$ des molécules HLA de classe II. Cette hypothèse a pu être infirmée par les expériences réalisées par D. Charron qui, par électrophorèse en double dimension, mettent en évidence cette chaîne dans les lysats cellulaires des patients [17].

L'ensemble de ces résultats conduisit à rechercher les anomalies au niveau des ARN messagers cytoplasmiques des cellules (au repos ou après induction blastique par la PHA). C. de Préval et coll. [19] utilisant les diverses sondes (ADNc) correspondant aux chaînes $\alpha$ et $\beta$ de spécificités DR, $\mathrm{DQ}$ et DP ont montré, par analyse en Northern blot, l'absence des ARN messagers cytoplasmiques codant pour les molécules HLA de classe II dans les cellules des patients. En revanche, ceux correspondant à la chaîne invariante étaient normalement trouvés. D'autre part, les ARN codant pour la chaîne lourde des molécules HLA de classe I étaient également présents, leur quantité variant d'un malade à l'autre,

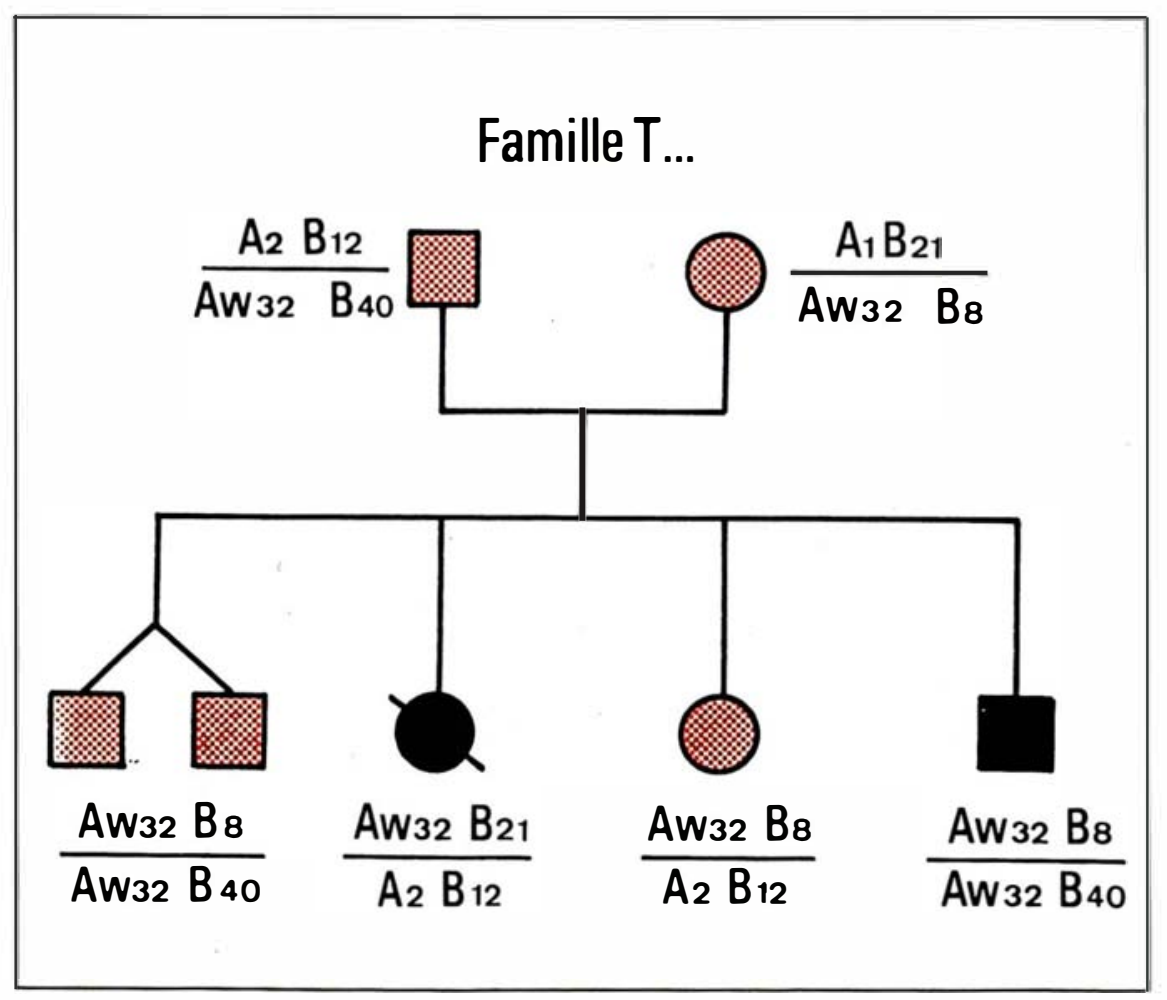

Figure 5. Arbre généalogique d'une famillo comportant deux enfants atteints ot illustrant la non-ségrégation do la maladio avec les haplotypes HLA ABC. 


\section{RÉFÉRENCES}

3. Segall M, Cairns JS, Dabl CA, et al. DNA and proteins studies of HLA Class II molecules : their relationship to $\mathrm{T}$ cell recognition. Immunol Rev $1985 ; 85$ : 129-34.

4. Kimball SE, Caligan JE. Structure of Class I major histocompatibility antigens. Top $\mathrm{Mol}$ Immunol 1983 ; 9 : 1-6.

5. Korman AJ, Boss JM, Spies T, Sorrentino R, Okada K, Strominger JL. Genetic complexity and expression of human class II histocompatibility antigens. Immunol Reviews $1985 ; 85: 45-50$.

6. Auffray C, Strominger JL. Molecular genetics of the human major histocompatibility complex. Advances in human genetics $1986 ; 15$ : 197-247.

7. Fellous M, Nir V, Wallach D, Merlin G, Rubinstein M, Revel M. Interferon dependent induction of mRNA for the major histocompatibility antigens in human fibroblasts and lymphoblastoid cells. Proc Natl Acad Sci USA $1982 ; 79: 3032-86$.

8. Griscelli C, Durandy A, Virelizier J, et al. Impaired cell to cell interaction in partial immunodeficiency with variable expression of HLA antigens. In : Seligmann M, Hitzig WH, eds. Primary Immunodeficiencies. Amsterdam : Eelsevier/North-Holland, 1980 : 499-503.

9. Touraine JL, Betuel H, Souillet G, Jeune $M$. Combined immunodeficiency disease associated with absence of cell surface HLA-A and B antigens. J Pediatr 1978 ; 93 : 47-51.

10. Schuurman RKB, Van Rood JJ, Vossen JM, Schellekens PTA, Feltkamp-Vroom TM Visser HKA. Hypogammaglobulinemia and HLA gene products. Pediatr Res $1978 ; 12: 71$. (Abstract.)

11. Touraine JL, Betuel H, Immunodeficiency diseases and expression of HLA antigens. Human Immunology 1981 ; 2 : 147-53.

12. Hadam MR, Dopfer R, Peter HH, Niethammer D, Congenital agammaglobulinemia associated with lack of expression of HLA Dregion antigens. In : Griscelli C, Vossen J, eds. Progress in Immuradficiong Research and Therapy. Amsterdam : Excerpta Medica, 1984; 19-26.

13. Kuis W, Roord JJ, Zegers BJM, at al. In : Touraine JL, Gluckman E, Griscelli C, eds Bone Marrow Transplantation in Europe II. Ams terdam : Excerpta Medica, 1981 : 201 p.

14. Durandy A, Virelizier JL, Griscelli C. Enhancement by interferon of membrane HLA antigens in patients with combined immunodeficiency with defective HLA expression. Clin Exp Immunol 1983 ; 52 : 173-8.

15. Lisowska-Grospierre B, Durandy A, Virelizier JL, Fischer A, Griscelli C. Combined immunodeficiency with defective expression of

HLA : modulation of an abnormal HLA synthesis and functional studies. Birth Defects parallèlement à celles des protéines dont ils dirigent la synthèse (figure 6).

L'ensemble de ces résultats suggère très fortement que l'anomalie génétique observée est liée à un défaut d'un gène intervenant dans la régulation de l'expression des gènes de classe II du CMH. Ces résultats montrent de plus que ce gène est situé en dehors du CMH et agit en trans.

\section{Discussion}

Le déficit immunitaire combiné sévère caractérisé par un défaut de synthèse des molécules d'histocompatibilité de classe II lié à un défaut génétique autosomique récessif, vient confirmer l'importance que jouent ces molécules dans les réponses immunes. Cette affection est un modèle permettant d'une part l'étude des interrelations cellulaires impliquées dans les fonctions immunologiques et d'autre part l'étude de l'expression des gènes de structure et de régulation correspondant aux HLA de classe II. Les anomalies fonctionnelles rencontrées dans cette affection se résument en un défaut de réponse cellulaire et humorale aux antigènes résultant très probablement du défaut d'expression membranaire des molécules d'histocompatibilité de classe II. En effet aucune autre anomalie membranaire n'a pu être mise en évidence dans les cellules lymphocytaires de ces malades, les marqueurs ou récepteurs normalement exprimés par les lymphocytes $\mathrm{T}$ activés (T9, TAC) étant notamment parfaitement détectables.

Le déficit immunitaire associé à un défaut de synthèse des molécules HLA de classe II paraît différent d'une autre affection décrite par Touraine et coll. $[9,11]$ et Schuurman et coll. [10], affection entrầnant un déficit immunitaire profond responsable du décès dans les premières années de vie. Le déficit est ici caractérisé par un défaut apparemment isolé de l'expression membranaire des molécules HLA de classe I. Plus récemment, Payne et coll. [20] ont décrit un défaut d'expression des HLA de classe I avec expression normale des HLA de classe II chez des sujets ne présentant ni déficit immunitaire fonctionnel, ni susceptibilité particulière aux infections. Il demeure en fait une certaine ambiguité puisqu'il est possible que les malades observés par Touraine et Schuurman aient pu correspondre à des défauts de synthèse des HLA de classe II non détectés à un moment où les moyens d'investigation étaient encore modestes. Chez tous les malades que nous avons étudiés, l'expression membranaire, le niveau de synthèse intracytoplasmique et la quantité d'ARN messagers correspondant à la chaîne lourde des HLA de classe I sont diminués. Ce défaut partiel de l'expression des molécules HLA de classe I pourrait être secondaire aux anomalies primitives de la synthèse des molécules HLA de classe II responsables du déficit immunitaire fonctionnel. Cette interprétation est surtout fondée sur le fait que l'expression des molécules HLA de classe I peut être corrigée par les interférons. Cette situation n'est pas sans rappeler celle observée chez le nouveau-né humain normal où l'expression des molécules HLA de classe I est diminuée et paraît corrélée à une diminution des capacités de sécrétion d'interféron par les lymphocytes $\mathrm{T}$ [21]. L'importance relative du profond défaut de l'expression des molécules HLA de classe II et du défaut partiel d'expression de celles de classe I dans la susceptibilité aux infections virales est intéressante à considérer. Chez l'homme, les moyens de défense antivirale dépendent de l'anticorps lui-même, de la cytotoxicité anticorps-dépendante (ADCC) et de la cytotoxicité spécifique T (CTL). Dans cette affection, les deux premiers moyens de défense antivirale manquent, du fait de l'anomalie de la production d'anticorps. On est frappé de constater une fréquence et une gravité des infections virales supérieures à celles observées chez les sujets atteints d'un défaut complet, mais isolé, de la production d'anticorps comme l'agammaglobulinémie liée au chromosome X. Ceci suggère 


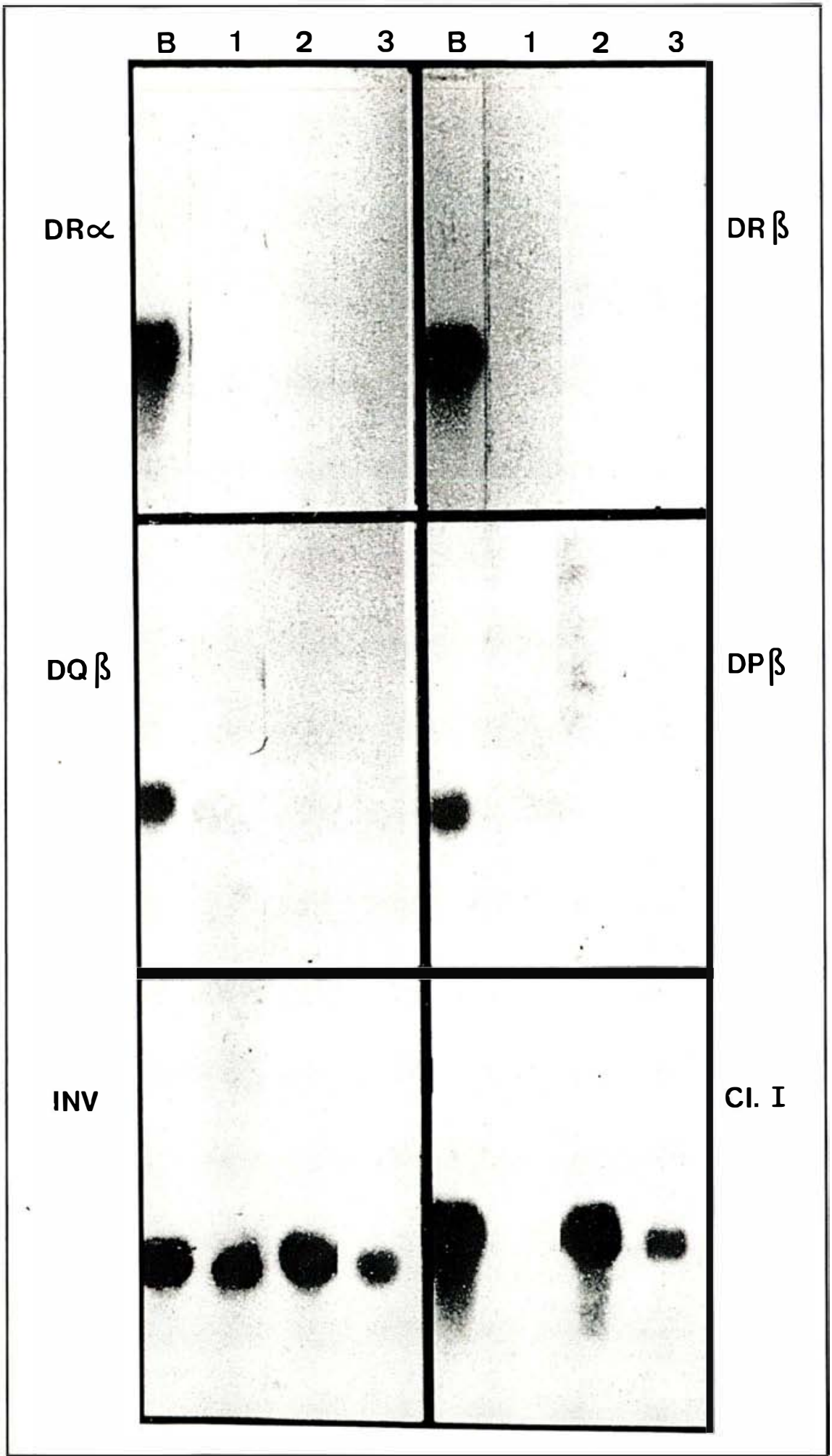

Figure 6. Analyse par " northem blot" des ARN obtenus à partir des blastes PHA des pationts dóficionts on HLA do classo II (lignes 1-3) ot d'un sujet témoin (B). Les sondes $d^{\prime} A D N$ spécifiques de : $D R \alpha, D R \beta, D Q \beta$ et $D P \beta$, de la chaîne invariante (INV) et de la chaîne lourde de HLA de classe I (CI. I) marquées au $p^{32}$, ont été utilisées pour détecter les transcrits ARN spécifiques de ces gènes, selon les méthodes décrites réf. [19].

$m / s n^{\circ} 1$ vol. 3 , janvier 87 qu'au cours du défaut d'expression des molécules HLA de classe II, le troisième moyen de défense anti-virale, l'activité CTL, est également perturbé.

Sur le plan génétique, la description des anomalies responsables du défaut de synthèse des molécules de classe II suggère l'existence de gènes de régulation de l'expression des gènes de classe II du CMH. En effet, dans cette affection, le défaut touche l'expression de nombreux gènes situés sur le chromosome 6 , alors qu'aucune anomalie grossière au niveau de l'ADN n'a pu y être détectée. De plus, la non coségrégation du trait génétique et des haplotypes parentaux fait apparaître l'absence de liaison génétique entre le déficit observé et les gènes du CMH. Le mécanisme d'une telle anomalie de régulation n'est pas encore connu et peut être transcriptionnel ou post-transcriptionnel. Il est intéressant de rappeler que tous les gènes HLA de classe II possèdent, chez l'homme comme chez la souris, des séquences non codantes appelées class II Boxes [22] situées à leur extrémité 5', séquences réceptrices des signaux de régulation. Dans l'affection que nous avons décrite, l'anomalie d'expression des gènes HLA de classe II du CMH n'est pas levée par l'interféron $\gamma$. Il semble donc que l'induction de l'expression des gènes de cette région du $\mathrm{CMH}$ par l'interféron nécessite un intermédiaire, ici anormal, le fonctionnement du gène de régulation. On ne peut cependant pas à ce stade de nos connaissances déterminer si la régulation des gènes HLA de classe II, ici anormale, est de type positif (absence d'un activateur nécessaire à l'induction) ou de type négatif (analogue à celle décrite chez les procaryotes). En effet, si l'induction des gènes HLA de classe II dépendait d'une inhibition d'un signal négatif, l'absence de cette inhibition ou son défaut dû à une anomalie génique pourrait également aboutir à un défaut de la transcription des gènes de classe II. Enfin, l'anomalie pourrait être post- 


\section{RÉFÉRENCES}

16. Griscelli C, Fischer A, Grospierre B, at al. Clinical and immunological aspects of combined immunodeficiency with defective expression of HLA antigens. In : Griscelli C, Vossen J, eds. Progress in Immunodeficiency Research and Therapy I. Amsterdam : Excerpta Medica, 1984 : 19-26.

17. Lisowska-Grospierre B, Charron DJ, De Préval C, Durandy A, Griscelli C, Mach B. $A$ defect in the regulation of major histocompatibility complex class II gene expression in human HLA-DR negative lymphocytes from patients with combined immunodeficiency syndrome. J Clin Invest $1985 ; 76$ : 381-5.

18. Marcadet A, Cohen D, Dausset J, Fischer A, Durandy A, Griscelli C. Genotyping with DNA probes in combined immunodeficiency syndrome with defective expression of HLA. $N$ Engl $J$ Med 1985 ; $31-2$ : 1287-92.

19. De Préval C, Lisowska-Grospierre B Loche M, Griscelli C, Mach B. A trans-acting class II regulatory gene unlinked to the MHC controls expression of HLA class II genes. Nature 1985 ; 318 : 291-5.

20. Payne R, Brodsky FM, Peterlin BM, Young LM. Bare lymphocytes without immunodeficiency. Human Immunology $1983 ; 6$ : 219-27.

21. Wagasuki N, Virelizier JL. Defective IFN gamma production in the human neonate $\mathrm{I}$ : Dysregulation rather than intrinsic abnormality. J Immunol 1985 ; 134 : 167-71.

22. Saito H, Mali RA, Clayton LK, Tonegawa $\mathrm{S}$. Complete primary structures of the $\mathrm{E} \alpha$ chain and gene of the mouse major histocompatibility complex. Proc Natl Acad Sci USA $1983 ; 80$ : 5520-4

23. Gladstone $P$, Pious D. Stable variants affecting B cell alloantigens in human lymphoid cells. Nature 1978 ; 271 : 459-61.

24. Levine F, Pious D. Revertants from the HLA class II regulatory mutant 6.1 .6 : Implications for the regulation of Ia gene expression. J Immunol 1984 ; 132 : 959-63.

25. Accolla RS, Human B. Cell variants immunoselected against a single Ia antigen subset have lost expression of several Ia antigen subsets. $J$ Exp Med 1983 ; 157 : 1053-8.

26. Accolla RS, Carra G, Guardiola J. Reactivation by a trans-acting factor of human major histocompatibility complex Ia gene expression in interspecies hybrids between an Ia-negative human B-cell variant and an Iapositive mouse B-cell lymphoma. Proc Natl Acad Sci USA 1985 ; 82 : 5145-9.

27. Salter RD, Alexander J, Levine F, Pious D, Cresswell P. Evidence for two trans-acting genes regulating HLA class II antigen expres- transcriptionnelle, notamment due à une instabilité des transcrits. Les anomalies décrites dans le défaut de synthèse des molécules HLA de classe II sont à comparer aux résultats expérimentaux obtenus par Pious et coll. [23, 24] et Accolla et coll. [25, 26] par irradiation et immunosélection de lignées lymphocytaires B humaines. Les variants ainsi obtenus sont caractérisés par un défaut de synthèse des molécules HLA de classe II. Il est intéressant de constater que ces variants ont des caractéristiques voisines de celles des mutants génétiques que nous décrivons : défaut d'expression des gènes HLA de classe II sans anomalie décelable des gènes de structures, intégrité des ARN messagers de la chaîne invariante et défaut de synthèse des ARN messagers correspondant à l'ensemble des molécules HLA de classe II. Les études réalisées sur ces modèles expérimentaux conduisent à envisager l'existence d'un ou de plusieurs gènes de régulation agissant en trans. Accolla et coll. [26] montrent que les mutants classe II négatifs peuvent réexprimer les gènes silencieux après fusion somatique avec des cellules de lymphomes B murins. Pious et coll. [24] font des constatations comparables et, très récemment, suggèrent, grâce à des expériences de complémentation, l'existence d'au moins deux gènes de régulation différents [27].

L'étude du défaut de synthèse des molécules HLA de classe II a ainsi permis d'apporter de nouveaux arguments en faveur de l'existence d'un ou plusieurs gènes capables de contrôler l'expression et la synthèse des molécules HLA de classe II. Ce(s) gène(s) doit(doivent) être actifs dans toutes les cellules synthétisant les molécules HLA de classe II puisque toutes, d'origine médullaire ou autre, sont affectées par la maladie. L'isolement de ces gènes permettra de caractériser l'affection et de prouver leur intervention dans cette maladie en corrigeant par transfert génique les anomalies cellulaires observées

\section{Summary}

Major histocompatibility complex (MHC) class II antigens are highly polymorphic cell surface glycoproteins that play a central role in the immune responses. An inherited severe combined immunodeficiency (SCID) in man is characterized by an absence of membrane expression of HLA (human leucocyte antigen) class II molecules and a decreased expression of HLA classe I molecules on peripheral blood leucocytes. These patients have a profound defect of antibody production and of cell-mediated immunity leading to infections which are often lethal. Synthesis of all HLA class II molecules was found absent and synthesis of HLA class I molecules decreased in patient leucocytes and PHA-induced blasts. On the RNA level, an absence of messenger RNAs for the $\alpha$ and $\beta$ chains of HLA-DR, $\mathrm{DQ}$ and DP was observed, indicating a global defect in the expression of all HLA class II genes. Family studies have established that the genetic defect does not segregate with the MHC. Since the structural genes coding for class II molecules do not seem to be affected, we conclude that a trans-acting regulatory gene, controlling the expression of HLA class II genes but unlinked to the $\mathrm{MHC}$, is affected in these patients.

\section{TIRÉS À PART}

B. Lisowska-Grospierre : Inserm U. 132, hôpital Necker-Enfants Malades, 149, rue de Sèvres, 75015 Paris. 